\title{
A Centralized Win-Win Cooperative Framework for Wi-Fi and 5G Radio Access Networks
}

\author{
A. Raschellà $\mathbb{D}^{1},{ }^{1}$ O. Aldhaibani, ${ }^{1}$ S. Pizzi, ${ }^{2}$ M. Mackay, $^{1}$ F. Bouhafs, ${ }^{3}$ G. Araniti, ${ }^{2}$ Q. Shi, ${ }^{1}$ \\ and M. C. Lucas-Estañ ${ }^{4}$ \\ ${ }^{1}$ School of Computer Science and Mathematics, Liverpool John Moores University (LJMU), James Parsons Building, Byrom Street, \\ L33AF, Liverpool, UK \\ ${ }^{2}$ DIIES, University "Mediterranea" of Reggio Calabria, Via Graziella, Loc. Feo di Vito, 89100 Reggio Calabria, Italy \\ ${ }^{3}$ School of Engineering and IT, University of New South Wales Canberra, Building 15, Northcott Drive, Campbell, \\ ACT 2600, Australia \\ ${ }^{4}$ UWICORE Laboratory, Universidad Miguel Hernández de Elche (UMH), Avda. de la Universidad s/n Edificio Quorum V 03202 , \\ Elche, Spain
}

Correspondence should be addressed to A. Raschellà; a.raschella@ljmu.ac.uk

Received 2 March 2021; Revised 29 July 2021; Accepted 25 August 2021; Published 8 September 2021

Academic Editor: Hui Cheng

Copyright (C) 2021 A. Raschellà et al. This is an open access article distributed under the Creative Commons Attribution License, which permits unrestricted use, distribution, and reproduction in any medium, provided the original work is properly cited.

\begin{abstract}
Cooperation to access wireless networks is a key approach towards optimizing the use of finite radio spectrum resources in overcrowded unlicensed bands and to help satisfy the expectations of wireless users in terms of high data rates and low latency. Although solutions that advocate this approach have been widely proposed in the literature, they still do not consider a number of aspects that can improve the performance of the users' connections, such as the inclusion of (1) cooperation among network operators and (2) users' quality requirements based on their applications. To fill this gap, in this paper we propose a centralized framework that is aimed at providing a "win-win" cooperation among Wi-Fi and cellular networks, which takes into account 5G technologies and users' requirements in terms of Quality of Service (QoS). Moreover, the framework is supported by smart Radio Access Technology (RAT) selection mechanisms that orchestrate the connection of the clients to the networks. In particular, we discuss details on the design of the proposed framework, the motivation behind its implementation, the main novelties, its feasibility, and the main components. In order to demonstrate the benefits of our solution, we illustrate efficiency results achieved through the simulation of a smart RAT selection algorithm in a realistic scenario, which mimics the proposed "win-win" cooperation between Wi-Fi and cellular 5G networks, and we also discuss potential benefits for wireless and mobile network operators.
\end{abstract}

\section{Introduction}

The wireless communication sector is witnessing a significant and ongoing increase of devices connecting to the Internet that might lead it to reshape the way services will be provided to clients. The use of portable computing devices such as smartphones, tablets, and laptops is dramatically increasing, and their popularity is also related to the emergence of innovative mobile applications and online services, which are driving the demand for more reliable wireless Anything, Anyone, Anytime, Anyplace (4A) communication connectivity [1]. In addition, modern mobile devices and innovative applications will be part of the $5^{\text {th }}$ Generation (5G) of mobile networks. The 3GPP is currently standardizing New Radio (NR), the air interface of the 5G system [2]. It mainly promises data rates of gigabits per second everywhere, significant reduction of latency, increase of system capacity by 100-1000 times over that of the current Long Term Evolution $4^{\text {th }}$ Generation (4G/LTE) standard, and 10-100 times higher density of the connected devices per region [3]. Wireless network operators are, therefore, challenged to introduce new solutions and mechanisms to 
optimize the use of the finite radio spectrum resource and, at the same time, to satisfy their clients by providing $4 \mathrm{~A}$ connections efficiently.

On the other hand, effective solutions must be designed considering that the use of the unlicensed spectrum bands is currently a key common approach adopted by most wireless technologies. It is well known, for instance, how unlicensed spectrum bands have been widely used by Wi-Fi networks. Specifically, the 2.4 and $5 \mathrm{GHz}$ Industrial, Scientific, and Medical (ISM) bands are used by the 802.11 $\mathrm{a} / \mathrm{b} / \mathrm{g} / \mathrm{n} / \mathrm{ac} / \mathrm{ax}$ standards, and the $60 \mathrm{GHz}$ millimeter wave (mmWave) band is considered by 802.11 ad/aj. Even cellular operators have started to seriously consider the use of unlicensed spectrum in order to optimize the capacity of cellular networks. In this context, LTE-unlicensed (LTE-U) technology has been the first step towards this approach and initiated as part of LTE Release 13 to enable access to both the licensed and unlicensed spectrums through a common infrastructure [4]. mmWave technology is an attractive solution also here. In fact, it allows operators to increase the capacity of $4 \mathrm{G} / \mathrm{LTE}$ networks towards the one promised for $5 \mathrm{G}$ systems. However, mmWave signals are vulnerable against foliage, rain, and humidity absorption and can only reach out to a few kilometers [5]. Therefore, the use of the sub$6 \mathrm{GHz}$ spectrum will still be essential for $5 \mathrm{G}$ systems due to the more favourable propagation characteristics and also to the possibility to have $5 \mathrm{G}$ systems backward compatible with current technologies operating in these bands. In fact, unlicensed access is being accepted as one of the most significant solutions to improve the resource availability and system scalability in $5 \mathrm{G}$ and beyond $5 \mathrm{G}$ (B5G) [6], and $5 \mathrm{G} \mathrm{NR}$ is being designed to support operation in unlicensed bands through a technology referred to as NR-based access to unlicensed spectrum (NR-U), which enables the operation of NR in the sub-6 GHz unlicensed bands [2].

In addition, other wireless technologies, such as Internet of Things (IoT) systems, Direct-to-Direct (D2D) communications, and Bluetooth, are increasingly used to connect devices with each other and the Internet using the unlicensed sub- $6 \mathrm{GHz}$ bands $[7,8]$. In a nutshell, it is undoubted that unlicensed bands are more and more overcrowded by completing wireless technologies. Especially Wi-Fi, which represents a cheaper and more diffuse alternative for many wireless users, service providers, and network operators, is seriously overloaded.

Staying with Wi-Fi and cellular networks, which undoubtedly represent the most ubiquitous and commonly used wireless technologies, we argue that although many radio resource management solutions can be found in the literature, which achieve encouraging performance results [9-11], they lack efficient coordination in the unlicensed bands that might be a key solution to optimize the use of bandwidth and provide adequate $4 \mathrm{~A}$ connections among both Wi-Fi and cellular networks users. The main problem related to these technologies that should be addressed through efficient coordination is that cellular networks selfishly occupy bands commonly used by Wi-Fi networks, generating interference with Wi-Fi. For instance, in [9], we have illustrated how Wi-Fi networks are located in environments where certain Access Points (APs) can be exposed to external interference from inaccessible sources. Moreover, the overcrowding of Wi-Fi networks forces mobile 4G/5G wireless Users Equipment (UE) to renounce connecting to $\mathrm{Wi}-\mathrm{Fi}$ and to prefer the use of cellular networks. This raises the necessity for better coordination between $\mathrm{Wi}-\mathrm{Fi}$ and cellular networks to benefit both technologies and their wireless users.

In this context, this paper is aimed at addressing the following novel contributions:

(i) First, we advocate a framework to address the spectrum congestion problem in unlicensed bands, which simultaneously benefits the satisfaction of both Wi-Fi and 5G users through cooperation between these networks that can be managed by centralized controllers

(ii) Secondly, we propose to guarantee a "win-win" cooperation between $\mathrm{Wi}-\mathrm{Fi}$ and $5 \mathrm{G}$ networks through a RAT selection algorithm implemented in the framework able to orchestrate the connection of the users with the most suitable RAT based on their ongoing application and profile customization gathered in the central controllers

We have opted for the "win-win" notation in order to highlight how our approach provides performance enhancements for both technologies, 5G and Wi-Fi networks, through cooperation. To demonstrate the efficiency of our solution, we provide a performance analysis developed through a system-level simulator, which implements the framework managed by a centralized controller in a realistic scenario to mimic how the "win-win" cooperation benefits both $5 \mathrm{G}$ and Wi-Fi networks.

The paper is organized as follows. Section 2 provides a detailed analysis of the limitations that standard-based RAT selection faces due to the lack of cooperation, the current works addressing coexistence through multi-RAT selection together with their limitations, and further details on our novel contributions. Then, in Section 3, we discuss the advocated cooperative framework to highlight its feasibility with regard to the upcoming $5 \mathrm{G}$ systems and its main components. Afterwards, in Section 4, we present the details and the performance evaluation results of the RAT selection algorithm, which orchestrates users' connections guaranteeing the "win-win" cooperation addressed by our framework through the most suitable RAT based on user requirements and customized profiles. Final conclusions are given in Section 5 .

\section{Coordination between Wi-Fi and 5G Networks}

2.1. Current Limitations. Currently, dual-interface devices can switch connection from cellular to Wi-Fi networks and vice versa. For instance, the cellular network can be offloaded by exploiting the unlicensed Wi-Fi spectrum if the Signal to Interference plus Noise Ratio (SINR) perceived 


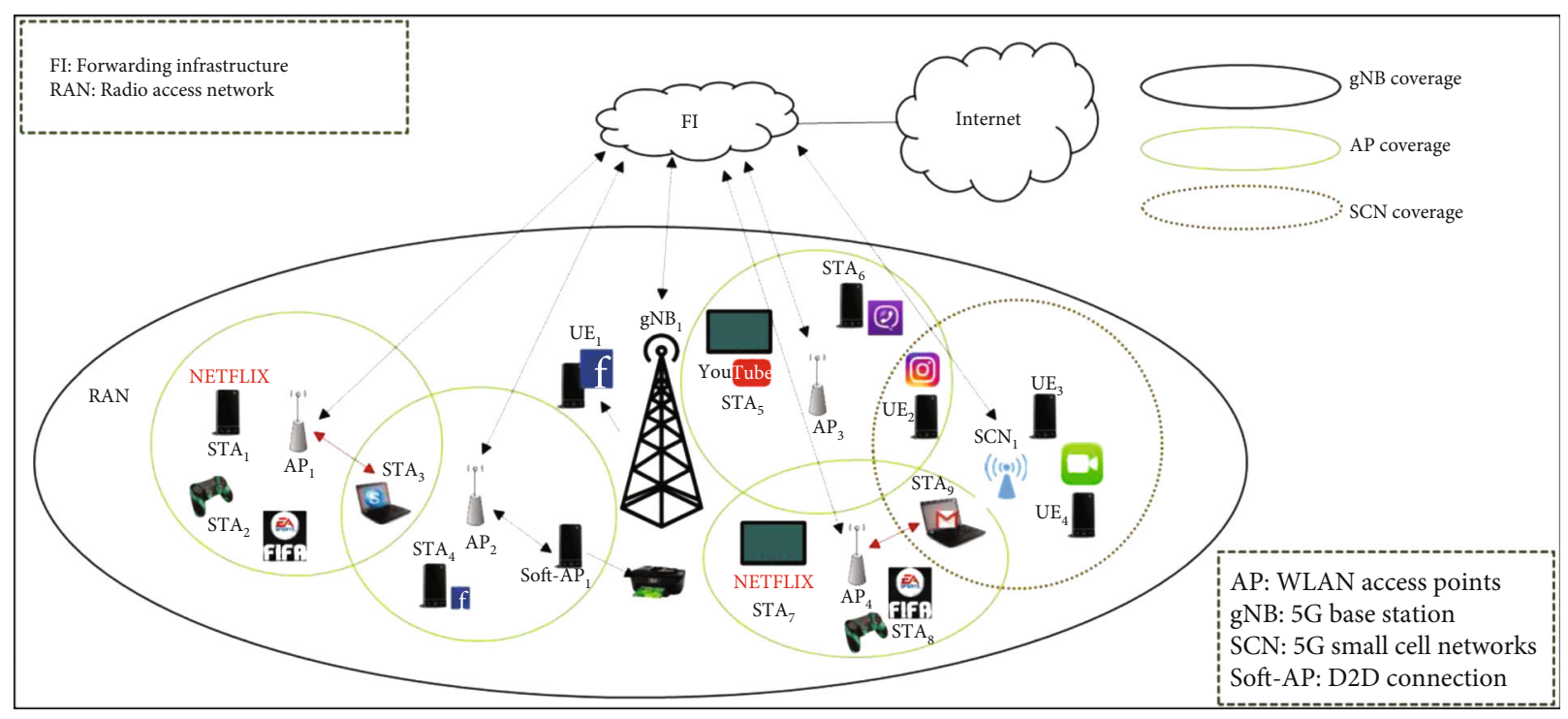

FIgure 1: Radio Access Technology standard approach.

by the UE is lower than a certain threshold [12]. Moreover, UEs can prioritize connections to uncongested Wi-Fi networks, even if the SINR experienced through the cellular network is above such a threshold. Hence, the connectivity with respect to the common scheme behind cellular/Wi-Fi network selection corresponds to either the Wi-Fi network if prioritized by the UE or the network providing a sufficient SINR. Furthermore, in the specific case of Wi-Fi, an AP selection approach for native Wi-Fi stations only (STAs), such as laptops, is usually based on the best Received Signal Strength Indicator (RSSI) as recommended by the IEEE 802.11 standards [9]. Therefore, standard network access approaches suffer the following crucial problems: (1) they easily cause network congestion because the bandwidth offered by current $\mathrm{Wi}-\mathrm{Fi}$ is not sufficient to satisfy the increasingly demanding requests of UEs and STAs; (2) they penalize Wi-Fi networks because dual-interface UEs might congest them, even if such UEs can obtain a sufficient quality of connection through cellular networks; (3) they do not allow offloading of traffic from Wi-Fi to cellular networks that might alleviate $\mathrm{Wi}-\mathrm{Fi}$ congestion without disruption to UEs connected to cellular networks; (4) they do not consider users' applications as a factor, which could affect the overall network performance; (5) they neglect that, to date, leveraging Wi-Fi networks has not solved cellular operators' bandwidth problems.

Figure 1 illustrates an example of standard approaches, consisting of both Wi-Fi and cellular networks. Specifically, in the figure, we show a realistic scenario that includes $5 \mathrm{G}$ access nodes that share the radio spectrum with Wi-Fi networks, and we highlight the limitations that it will face due to the lack of cooperation. The Forwarding Infrastructure (FI) can be related to either Wi-Fi or mobile networks, depending on the access node connected to it. The Radio Access Network (RAN) includes, for instance, Wi-Fi Access Points (APs), 5G Small Cell Networks (SCN), 5G base stations (gNBs), and soft-APs in the case of Device-to-Device (D2D) connections. For example, $\mathrm{STA}_{9}$ in the figure is allowed to connect only to $\mathrm{AP}_{4}$ although it is also in the area covered by $\mathrm{SCN}_{1}$. Moreover, from the figure, we can note $\mathrm{STA}_{7}$ and $\mathrm{STA}_{8}$ are connected to $\mathrm{AP}_{4}$ watching a video and playing an online game, respectively, thus requiring high data rates. This configuration could not allow satisfactory connection to $\mathrm{STA}_{9}$ trying to check e-mail, due to the high bandwidth needed for video streaming and online gaming. On the other hand, if $\mathrm{STA}_{9}$ was not bounded by the standard approach, it might connect to a currently unauthorized $\mathrm{SCN}$, i.e., $\mathrm{SCN}_{1}$ in the figure, reaching a sufficient quality for a low data rate application and without disruption to the UEs in $\mathrm{SCN}_{1}$. Therefore, it is clear in this example how cooperation between $\mathrm{Wi}-\mathrm{Fi}$ and $5 \mathrm{G}$ networks is essential to optimize the users' connections and alleviate the congestion problem.

2.2. Solutions Addressing Coexistence between Wi-Fi and Cellular Networks. Some works in the literature have proposed approaches to enable coordination among wireless users through harmonious coexistence between Wi-Fi and cellular networks in the unlicensed spectrum trying to address the limitations found in works that do not consider cooperation [9-11]. Some papers use techniques such as Clear Channel Assessment (CCA), Listen-Before-Talk (LBT), or Duty-Cycle Muting (DCM), meaning that a mobile device can transmit only when no ongoing transmission is observed for a specified period [13-16]. However, these techniques face challenging issues as mobile devices are required to sense a certain channel at a millisecond scale before a transmission. Moreover, the probability of a false alarm or error detection requires the implementation of efficient energy detection solutions. Finally, it has been demonstrated that in scenarios with a large number of Wi-Fi APs, these approaches can affect the performance of the coexistence system [15].

Other solutions can be found that try to solve these problems of access node allocation inefficiency through enhanced coexistence of Wi-Fi and cellular mobile networks $[1,12,17-21]$. Specifically, in [1], the authors studied the 


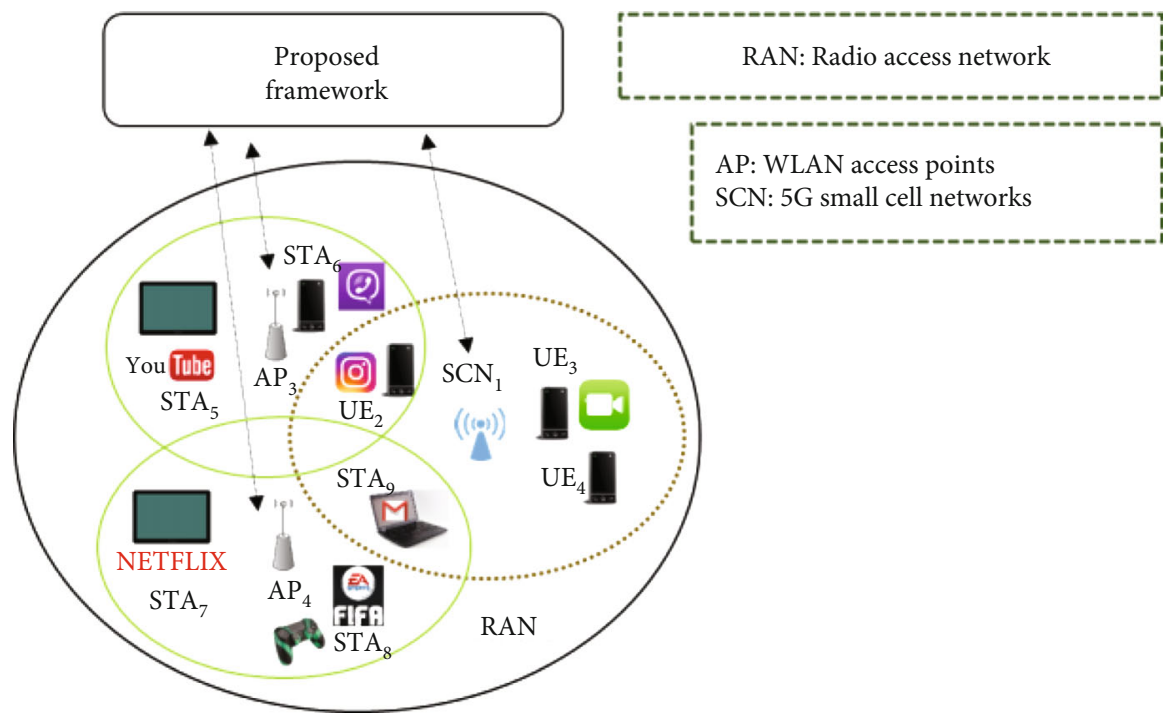

Figure 2: Proposed framework implemented on top of the RAN.

coexistence of LTE-U and Wi-Fi networks in a multichannel unlicensed spectrum scenario and proposed an algorithm based on Q-learning. In [12], the authors advocated an architecture that includes different RATs and allows the simultaneous use of cellular and Wi-Fi technologies. In $[17,18]$, the authors propose strategies for mobile networks and $\mathrm{Wi}-\mathrm{Fi}$ in heterogeneous networks to maximize the throughput. Other papers also address Quality of Service (QoS) requirements in terms of data bit rates of wireless users and Quality of Experience (QoE) defined through users surveys' results such as the works proposed in [19, 20], respectively. Finally, in [21], the authors presented 5GEmPOWER, a novel, programmable, and open-source Software-Defined Networking (SDN) platform for heterogeneous 5G RANs, guaranteeing simultaneous management of Wi-Fi and cellular networks. However, all these solutions still do not take into consideration a number of aspects that can further improve the performance of the users' connections. For instance, although all these papers advocate coexistence between cellular networks and $\mathrm{Wi}-\mathrm{Fi}$, they either do not aim to enhance Wi-Fi network performance and focus on 5 G UEs' benefit only, such as $[12,18,21]$, or focus only on LTE and Wi-Fi networks without considering 5G technologies, such as $[1,17,19,20]$. Additionally, a key limitation of all the solutions illustrated in this section is that they try to optimize objective key metrics in each access node, such as throughput, packet losses, and delay without taking into account the actual ongoing applications experienced by the wireless users. Note that the QoS requirements addressed in [19] are only represented by the data bit rates. On the other hand, in order to efficiently match a certain application to the most suitable access node, other key parameters are needed. For instance, an online call requires high QoS in terms of minimal delay rather than high data bit rates, which could be used to serve other users.

2.3. Novel Contributions. Motivated by the abovementioned limitations of CCA, LBT, DCM, and enhanced solutions based on coexistence in Wi-Fi and cellular mobile networks, in this paper, we analyse the potential for a novel cooperation approach between Wi-Fi and 5G networks through a centralized framework. Moreover, we propose and evaluate a multi-RAT selection algorithm based on users' requirements, which makes "win-win" the cooperation in terms of users' experiences conversely to the state-of-the-art. This algorithm is able to provide users with the most suitable RAT based on their ongoing application and customization, while optimizing the overall network satisfaction. In summary, the most important novelties and contributions of this paper, which is aimed at addressing the limitations of the abovementioned state-ofthe-art, can be summarised as follows:

(i) We advocate a cooperative solution implemented in a unique framework that, to the best of our knowledge, would simultaneously benefit both Wi-Fi and $5 \mathrm{G}$ users for the first time

(ii) We propose and discuss the benefits of a novel "winwin" cooperation that can be achieved through a RAT selection algorithm able to orchestrate the connection of wireless users to the most suitable RAT, based on their ongoing applications and customization profiles

\section{Advocated Cooperative Framework: Feasibility and Design}

The proposed framework, which is implemented on top of the RAN as illustrated in Figure 2, must be able to integrate and manage both 5G and Wi-Fi networks. Moreover, it must allow smart cooperation to efficiently guarantee QoS and QoE requirements to wireless users. Cooperative solutions need to be implemented in real-time and in smart controllers with a global view of users' devices and profiles, access nodes, and ongoing applications. Moreover, the controllers need to handle different technologies working in different 


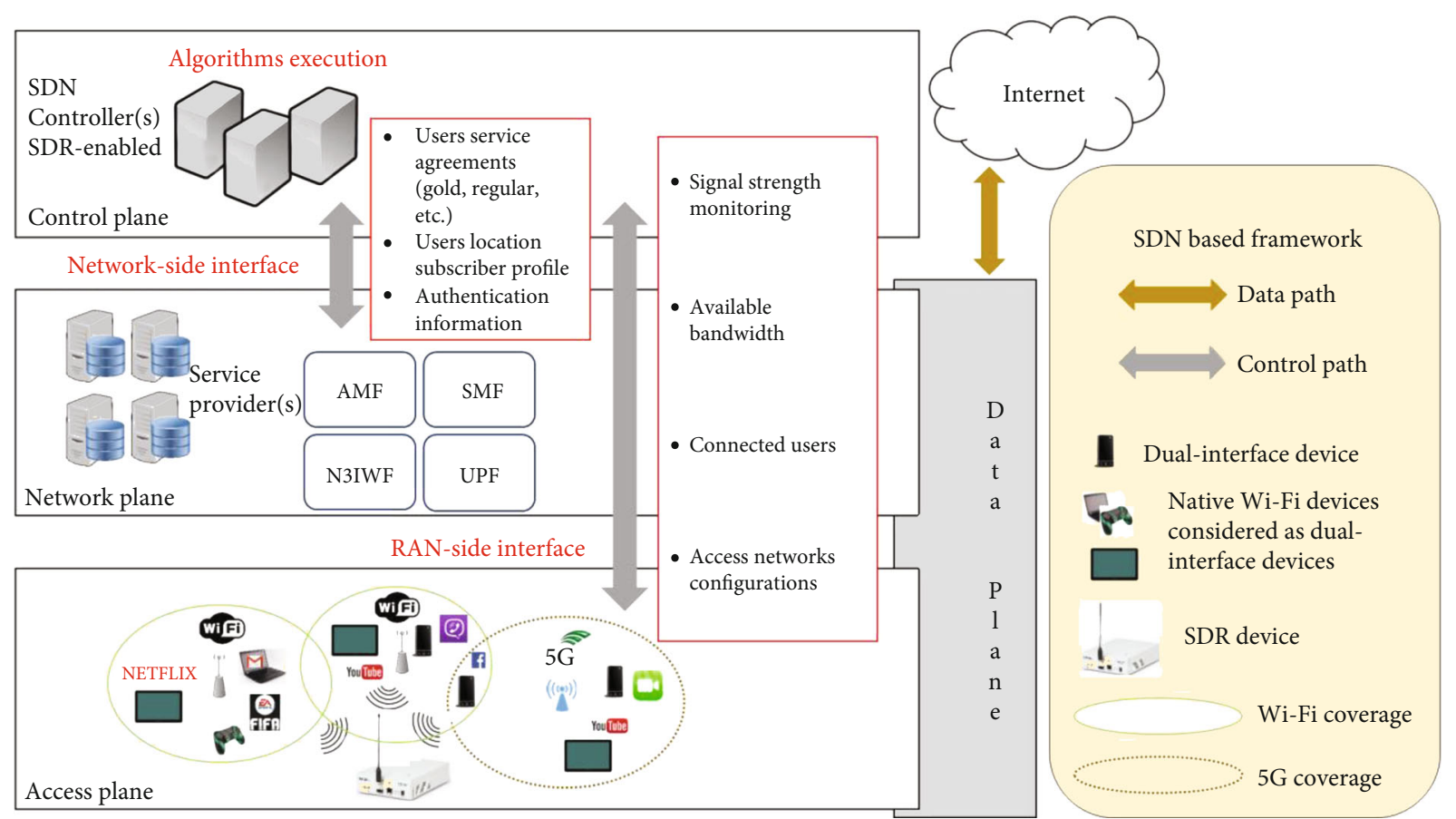

FIgURE 3: Proposed SDN-based interface framework.

spectrum bands, with various protocol stacks and vendor interfaces along with the monitoring capabilities. Therefore, we advocate the use of SDN to develop the proposed framework [22].

Note that $5 \mathrm{G}$ architectures already include new technologies to enable cooperation. In fact, UEs in the 5G systems will be able to use cellular and Wi-Fi technologies simultaneously [12]. Current 4G/LTE and 5G small base stations are easily deployed, reduce energy usage, and are becoming similar to Wi-Fi APs. Moreover, the interest of cellular operators in using unlicensed bands, as discussed in the Introduction, demonstrates how a deeper cooperation between cellular networks and Wi-Fi, which can leverage resources available in a whole system for all wireless users, is a realistic vision. Finally, note that [21] proposed an SDN-based platform able to simultaneously manage both $\mathrm{Wi}$ - Fi and $5 \mathrm{G}$ networks, and therefore, it validates the feasibility of the framework presented in this paper. Furthermore, note that our advocated framework proposes specific solutions to allow the efficient connection of users to both Wi-Fi and $5 \mathrm{G}$ networks, differently from the work presented in [21], which is aimed at benefiting only 5G UEs as we have mentioned in Section 2.2.

Moreover, we believe that SDN is the ideal tool for this framework because it does not require the deployment of a specific vendor's access nodes. Furthermore, it is able to provide southbound Application Programming Interfaces (APIs) to obtain key information for cooperative solutions, such as user preferences and radio environment status, and northbound APIs to implement smart algorithms in the wireless networks based on such information [21, 22]. Figure 3 illustrates our proposed SDN-based interface framework to allow full cooperation between $\mathrm{Wi}$-Fi and $5 \mathrm{G}$ networks. The proposed approach follows an SDN structure composed of different planes, which in our advocated framework are Data Plane, Access Plane, SDN-based Control Plane, and Network Plane. The description of these planes is provided in the following subsections.

3.1. Data Plane. The Data Plane consists of all the network elements located in Access and Network planes illustrated in Figure 3 and explained in the next subsections, for creating the legacy data packet transport network. Therefore, it includes switches, routers, and gateways.

3.2. Access Plane. The Access Plane is the RAN that includes 5G base stations and Wi-Fi APs. Moreover, we believe that this plane can leverage Software Defined Radio (SDR) technology, which is compatible with multiple RATs. Specifically, existing SDR hardware platforms are composed of an antenna, a multiband Radio Frequency (RF) module, and broadband Analog to Digital and Digital to Analog Converters (ADCs/DACs) and can be easily connected to central controllers able to manage them through software. For example, spectrum sensing functionalities relying on signals detected through an SDR platform can be implemented in the controller to monitor the interference level in a certain node. High levels of interference, for instance, make a certain node suitable only for users experiencing low data rate applications. An example of how the use of SDR can be leveraged to monitor interference can be found in [23], whereas a work that illustrates the benefits of controlling interference in a SDN-based framework to reduce their negative effects can be found in paper [9].

Moreover, note that in the advocated framework, native Wi-Fi devices such as laptops and smart TVs are considered 


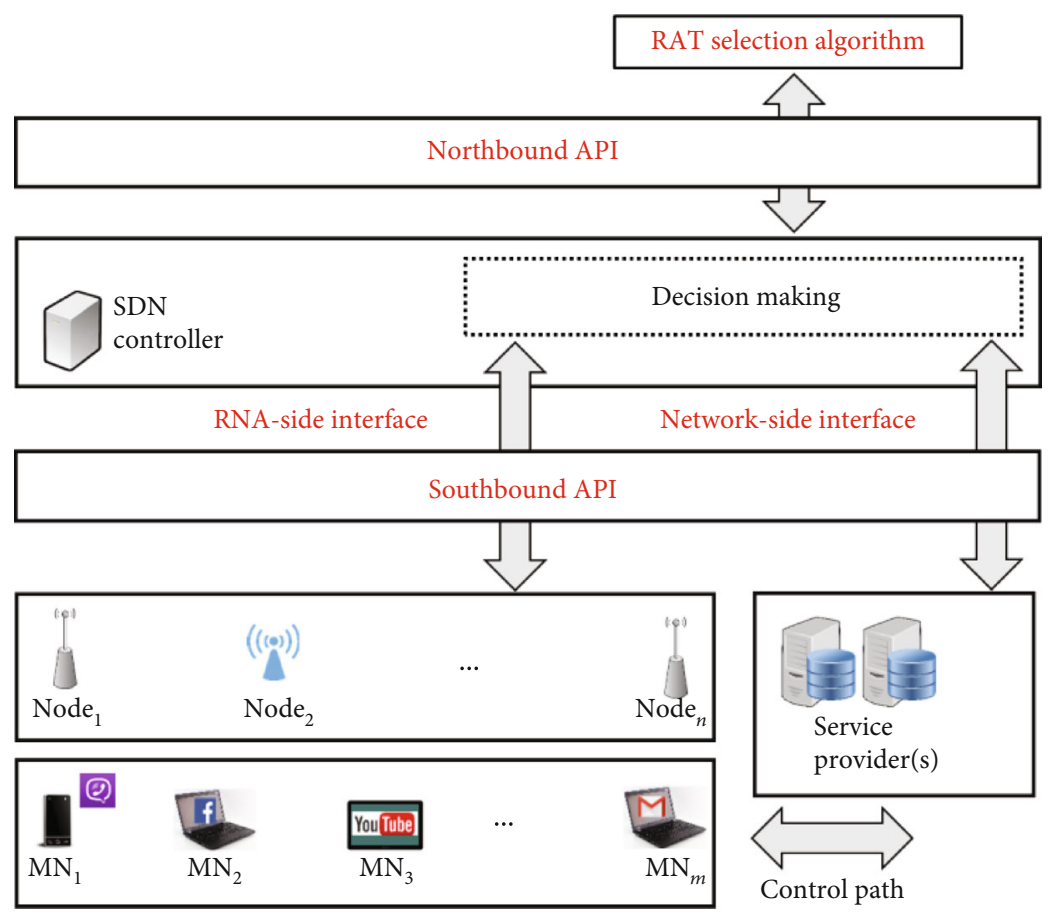

FIGURE 4: Simplified version of the proposed cooperative framework.

dual-interface devices in order to allow their connection to both Wi-Fi and cellular networks. Therefore, we consider that users have subscriptions with both services, which is a realistic assumption as LTE-enabled laptops are already available in the market and $5 \mathrm{G}$ laptops are foreseen to be in the market by the end of 2021 .

3.3. Network Plane. The Network Plane consists of different $\mathrm{Wi}-\mathrm{Fi}$ and 5G network complements, such as application servers and functionalities of the virtual 5G Core Network (5GC), which in turn include Access and Mobility Management Function (AMF), User plane function (UPF), Non3GPP InterWorking Function (N3IWF), and Session Management Function (SMF). In this plane, therefore, the profiles subscribed by the users can be stored. This information, together with those obtained from the Access Plane, will allow our framework to optimize specific Key Performance Indicators (KPIs), such as sufficient bandwidth for high definition(HD-) video streaming or low delay for VoIP applications.

3.4. SDN-Based Control Plane. The Control Plane includes the SDN-based controllers connected to the Network Plane and the Access Plane through RAN-side and network-side interfaces, respectively, as illustrated in Figure 3. Each controller, which can belong to a service provider or be shared among different service providers, is responsible for smart RAT selection strategies, which are based on several inputs received by both $\mathrm{RAN}$-side and network-side interfaces. Information provided by the RAN-side interface includes, among others, the signal strength received by each node at each RF channel, the noise, and the available bandwidth in each node. Information available in the network-side interface includes user service agreement and subscriber profiles.
SDN technology makes this approach feasible through the use of southbound and northbound Application Programming Interfaces (APIs), which are also central to other works found in the literature $[19,21,22]$. The southbound API is able to provide the controller with monitoring information, statistics, and events from all the network elements [22], whereas the northbound API enables different applications to program the wireless networks as desired, based on the information obtained through the southbound API. Moreover, the applications can be included and then removed as needed, and further algorithms can be implemented to properly orchestrate them. Typical applications are mobility management [21], AP radio parameter configuration (such as transmit power and RF channel selections), and horizontal/vertical handover [22]. A detailed example of the use of the information achieved from RAN-side and network-side interfaces and enabled by southbound and northbound APIs for a smart RAT selection algorithm will be provided in the next section. Specifically, our algorithm is able, through the northbound API, to orchestrate the connection of the users to the network based on the information achieved by the southbound API and then, supporting handover application.

\section{Illustrative Results}

Relying on the SDN-based framework previously presented, we now proceed with the description of a RAT selection algorithm based on user requirements and profile customization able to guarantee a "win-win" cooperation between $5 \mathrm{G}$ and Wi-Fi networks. Then, we provide the corresponding performance analysis. 
4.1. A RAT Selection Algorithm Based on Cooperation and User Customization. Figure 4 shows a simplified version of the framework illustrated in Figure 3 used to evaluate a RAT selection approach in a realistic scenario. The figure also illustrates the role of the northbound and southbound APIs. The framework is developed in an OPNET-based simulator and includes an SDN controller, which implements a smart RAT selection algorithm based on the information gathered from the network-side and RAN-side interfaces and processed in a decision-making module. From the figure, we can notice that the controller manages a set $N$ of access nodes equipped with different technologies of network operators that have agreed to cooperate and tightly merged in a unique wireless access network. Therefore, each node $N$ can be either a Wi-Fi AP or a gNB. Moreover, the framework provides connections to a set $M$ of Mobile Nodes (MNs), each one representing a dual-interface device able to join Wi-Fi and cellular networks, such as a smartphone or a $5 \mathrm{G}$ enabled laptop. The northbound API allows the handover application to program the wireless network based on the presented RAT selection algorithm, which uses the information obtained by the controller through the southbound API. Specifically, the southbound API provides the controller with monitoring information, statistics, and events from all the network elements, which can be used as input to our algorithm. Examples of well-known southbound APIs that can be found in the literature are OpenFlow protocol [24] and Cisco's OpenFlex interfaces [25]. Our framework relies on OpenFlow v1.3 over Transmission Control Protocol (TCP).

For each $\mathrm{MN} m \in\{1, \cdots,|M|\}$ that needs to be connected to a wireless network, the controller is able to gather the experienced SINR, available bandwidth, jitter, and delay through the RAN-side interface from each managed node $n \in\{1, \cdots,|N|\}$ that is allowed to provide connectivity to $m$. Note that, although the framework is simulated, the assumption of gathering the abovementioned information from the radio environment is realistic and can be performed in real time. For instance, the SDN-based Wi-5 architecture developed in the context of the Horizon 2020 (H2020) funded Wi-5 (What to do With the Wi-Fi Wild West) project is an example of a platform that includes a centralized controller able to collect such information in real time [22]. Moreover, the OPNET-based controller includes traffic detection software, which allows it to know the application being used by each $\mathrm{MN}$ for its connection to the most suitable access node. Finally, our simulator includes database service providers that give to the controller information related to the users' profile through the network-side interface, in order to achieve the current quality of connection negotiated by the users. For instance, a user might be subscribed to a mobile data plan that does not provide sufficient bandwidth for a high definition (HD) or $4 \mathrm{~K}$ video streaming on YouTube or Netflix.

After the collection of this information, they are matched by the controller to the corresponding Mean Opinion Scores (MOSs) that can be achieved by the MN $m$ from each access node $n$ able to provide connectivity. A MOS is a metric used to define the QoE that provides the human
TABle 1: Mean Opinion Score-MOS.

\begin{tabular}{lcc}
\hline MOS & Quality & Impairment \\
\hline 5 & Excellent & Imperceptible \\
4 & Good & Perceptible but not annoying \\
3 & Fair & Slightly annoying \\
2 & Poor & Annoying \\
1 & Bad & Very annoying \\
\hline
\end{tabular}

user's view of the quality of the network [19]. Specifically, the MOS is an arithmetic mean of all the individual scores achieved by the result of subjective tests and can range from 1 to 5 based on quality and impairment. The meaning of each score is illustrated in Table 1 in terms of quality and impairment. In detail, the qualities range from bad scored 1 , which represents a very annoying impairment, to excellent scored 5, which represents an imperceptible impairment. For instance, a user subscribed to a plan that does not provide sufficient bandwidth to watch an HD video streaming, therefore able to watch a Standard Definition (SD) H.264 video streaming, can reach an excellent quality from an access node able to guarantee a delay lower than $2 \mathrm{~s}$, a jitter lower than $20 \mathrm{~ms}$, an SINR of $20 \mathrm{dBm}$, and a minimum bandwidth of $900 \mathrm{kbps}$ [26]. The computation of the MOS based on the input considered in this paper is performed using an enhanced version of the Fuzzy Logic Control (FLC) method that has been proposed in [27] for Wi-Fi only network and which in this paper has been extended to work also for 5G networks. The explanation of the analytical details of this model is out of the scope of this paper and can be found in [27].

Finally, in the considered RAT selection, each time a new $\mathrm{MN}$ connects to the network, the controller chooses an access node based on the following tasks:

(i) Task 1. The controller collects the SINR, the available bandwidth, the jitter, and the delay experienced by the MN in each node that it manages through the RAN-side interface and through the network-side interface, and it collects the quality of connection negotiated by the MN.

(ii) Task 2. Based on the collected data, the controller selects the node guaranteeing the most suitable MOS to the new MN.

(iii) Task 3. The controller tries to guarantee a minimum value of the MOS that can be defined in each simulation. Therefore, each time a new MN connects to the network, the controller recomputes the MOSs for all the MNs of the managed networks in order to connect the ones experiencing a MOS below the established threshold due to the new connection, to a node providing at least the threshold value of the MOS when possible. The execution of the algorithm can trigger the handover of some $\mathrm{MNs}$ to other nodes. Note that this approach can be implemented in a real-time platform without perceptible 


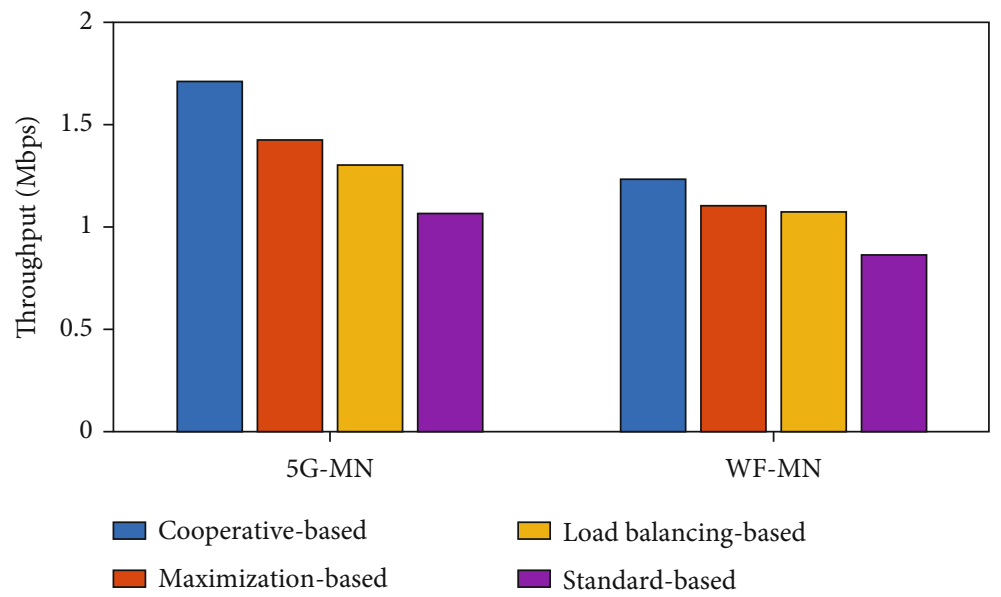

Figure 5: Performance results in terms of throughput.

disruption to the users' connections. For instance, as demonstrated in [22], the $\mathrm{Wi}-5$ architecture allows for seamless handovers to move users among nodes when needed, without noticeable data loss

4.2. Results. In order to evaluate our RAT selection implemented in the advocated cooperative framework, we developed the abovementioned OPNET-based simulator for a network composed of an SDN controller, 2 Wi-Fi 802.11n $2.4 \mathrm{GHz}$ APs, and $25 \mathrm{G} \mathrm{gNBs}$ that can belong to different network operators and use numerology $\mu=0$ and $20 \mathrm{MHz}$ channel bandwidth [28]. We assume that gNBs provide a maximum data rate of $100 \mathrm{Mbps}$. These nodes are randomly deployed in an area of $50 \times 50 \mathrm{~m}^{2}$ at a minimum distance of 40 meters among them representing a typical example of a dense environment [19]. We also simulated a set of 40 MNs requesting connection and uniformly distributed in the area. In order to evaluate our "win-win" cooperative framework, in this simulation, we consider that all the MNs can connect to both technologies: Wi-Fi and cellular networks. An $\mathrm{MN}$ can be either a $5 \mathrm{G}$ device or a Wi-Fi dual-interface device, called 5G-MN and WF-MN, respectively. Therefore, all devices are able to connect to both technologies based on our RAT selection strategy. Moreover, we assume that $8 \mathrm{MNs}$ run a VoIP G7.11 application and 32 MNs run H.264 video streaming with only 16 of them able to use the HD option based on their subscriptions. The minimum guaranteed MOS value in this simulation is 3 in order to guarantee at least the fair or slightly annoying option illustrated in Table 1.

To benchmark the performance of the RAT selection approach, we compare it against three reference strategies offering no cooperation discussed in this paper. The first is the RAT selection scheme based on IEEE standards. Therefore, in this case, we suppose that the controller gives a higher priority for all WF-MNs and 5G-MNs to connect to a Wi-Fi AP and let them switch to a cellular node when the received SINR is below the threshold of $3 \mathrm{dBm}$ [19]. The second strategy follows the work proposed in [29], which fairly distributes the MNs in the considered scenario based on a balanced load of the nodes in the network. Specif- ically, based on this solution, the controller associates each $\mathrm{MN}$ with the least loaded node, which provides a sufficient RSSI based on the QoS requirements. We consider this solution because it also targets a similar centralized approach relying on SDN. In the third approach, a central controller implements a RAT selection scheme that looks for all possible associations between MNs and nodes assigning each MN to either $\mathrm{Wi}$-Fi or $5 \mathrm{G}$ nodes in order to maximize the sum of logs of the MN's throughputs instead of the total MN's individual throughput [18]. Therefore, we demonstrate that our approach allows us to achieve better performance against common solutions that try to optimize the load balance of the network access nodes and the throughput experienced by the users in the network. The evaluation of our approach against these strategies focuses on the following performance metrics averaged for all 5G-MNs and WF-MNs after their connections to the corresponding access nodes: throughput, MOS, and delay.

Figures 5-7 illustrate the performance results averaged for both 5G-MNs and WF-MNs in the case of all the algorithms when all $40 \mathrm{MNs}$ are connected to the network. Specifically, Figure 5-7 show performance results for all the algorithms in terms of throughput, delay, and MOS, respectively. From the figures, we can see how our proposed cooperative solution improves the state of the art in terms of all the considered metrics and for both device types. In detail, from Figure 5, we can observe that when all the MNs are connected to the network, our RAT selection strategy based on collaboration outperforms the load balancing-based approach by around 24\%, the maximization-based one by approximately $17 \%$, and the standard-based solution by around $37 \%$, in the case of $5 \mathrm{G}-\mathrm{MNs}$. These percentages are $13 \%, 10 \%$, and $30 \%$ in the case of WF-MNs. Moreover, from Figure 6 , we can claim a reduction of delay through our approach of $63 \%, 41 \%$, and $77 \%$ with respect to the load balancing-based strategy, the maximization-based one, and standard-based solution, respectively, in the case of 5GMNs. This reduction is $60 \%$ for the load balancing-based strategy, $48 \%$ in the case of the maximization-based one, and $68 \%$ for the standard-based solution for WF-MNs. Finally, from Figure 7, we can observe gains in terms of 


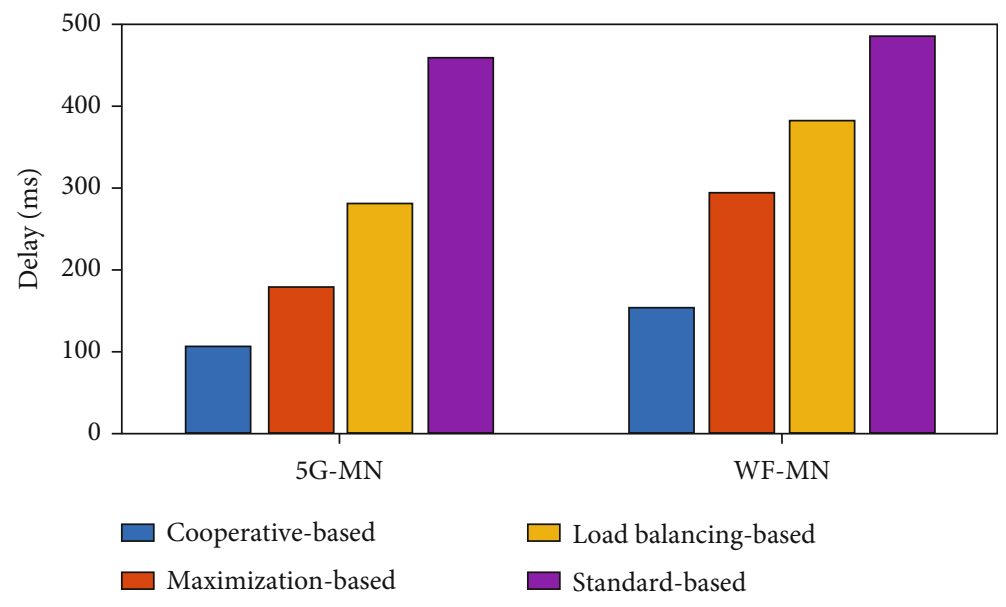

FIgURE 6: Performance results in terms of delay.

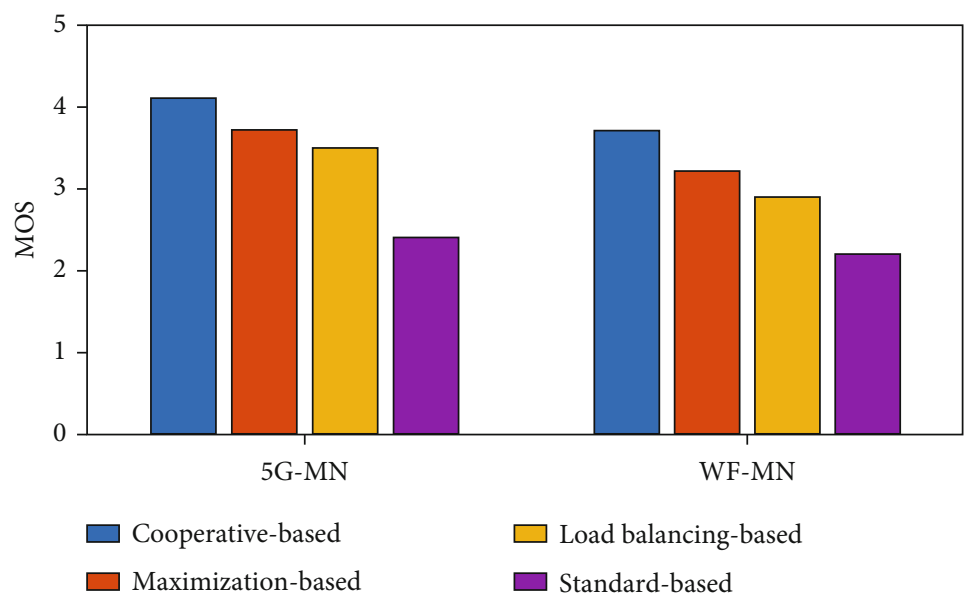

FIGURE 7: Performance results in terms of MOS.

MOS by $15 \%, 10 \%$, and $41 \%$ with respect to the load balancing-based strategy, maximization-based one, and standard-based solution, respectively, in the case of 5GMNs, and $22 \%, 14 \%$, and $41 \%$ with respect to the load balancing-based strategy, maximization-based one, and standard-based solution, in the case of WF-MNs. Therefore, these results demonstrate how using information on the radio environment and users' profiles, collected through an SDN-based controller connected to 5G and Wi-Fi RATs in a cooperative framework, provides performance enhancements for both technologies, especially compared to the standard approach.

Furthermore, we argue that the improvement we achieved through the proposed framework leads to a higher satisfaction of users that, in turn, can benefit telecommunication companies. In fact, today, in a highly competitive market, companies are interested to consider metrics to measure their financial profits, such as the so-called churn rate [30]. This metric represents the rate of customers, who cease their relationships with a certain company, and, therefore, is an indicator of customers' dissatisfaction due to poor performance and success on the part of their com- petitors [30]. Therefore, we believe that an improvement of the performance of the users' experiences, such as the one achieved through our cooperative framework, undoubtedly has a positive effect also in reducing the churn rate and, consequently, in the wireless network operators financial profits.

\section{Conclusions}

This paper has proposed a framework which is aimed at optimizing access to Wi-Fi and 5G networks through a full and "win-win" cooperation managed by centralized controllers. Specifically, the proposed framework is designed to enable cooperative mechanisms among different RATs, which can belong to either Wi-Fi or cellular network operators, to enhance users' connections. Moreover, the cooperative mechanisms are designed to provide the users with the most suitable RAT based on their ongoing application and profile customization. We have first identified the limitations of current standard RAT selections among cellular and $\mathrm{Wi}$-Fi radio access nodes and presented the novelties of this work compared to the state of the art. Then, we have described the proposed "win-win" cooperative framework, 
including the details of its feasibility and main components. Finally, we have provided a performance analysis developed through a system-level simulator, which implements a RAT selection strategy in a realistic scenario that mimics the full cooperation between $\mathrm{Wi}-\mathrm{Fi}$ and $5 \mathrm{G}$ networks managed by a shared centralized controller. Our results have demonstrated the benefits achieved for both technologies through our strategy compared to the current standard approach and another solution found in the state of the art. Finally, we have discussed how improvements in the user experience performance can also lead to a positive effect in the wireless network operators' financial profits.

\section{Data Availability}

Data supporting the conclusions of this work have not been made available because the license used to access the simulator designed for this study together with the achieved results belongs to Liverpool John Moores University.

\section{Conflicts of Interest}

The authors declare that they have no conflicts of interest.

\section{References}

[1] Y. Su, X. Du, L. Huang, Z. Gao, and M. Guizani, "LTE-U and Wi-Fi coexistence algorithm based on Q-learning in multichannel," IEEE Access, vol. 6, pp. 13644-13652, 2018.

[2] 3GPP specification Technical Report (TR) 38.913, "5G; study on scenarios and requirements for next generation access technologies," Rel, vol. 15, 2018.

[3] R. Bajracharya, R. Shrestha, R. Ali, A. Musaddiq, and S. W. Kim, "LWA in 5G: state-of-the-art architecture, opportunities, and research challenges," IEEE Communications Magazine, vol. 56, no. 10, pp. 134-141, 2018.

[4] 3GPP specification Technical Report (TR) 36.889, "Feasibility study on licensed-assisted access to unlicensed spectrum," Rel, vol. 13, 2015.

[5] N. Al-Falahy and O. Y. K. Alani, "Millimetre wave frequency band as a candidate spectrum for $5 \mathrm{G}$ network architecture: a survey," Physical Communication, vol. 32, pp. 120-144, 2019.

[6] S. Mumtaz, A. Jamalipour, H. Gacanin et al., "Licensed and unlicensed spectrum for future 5G/B5G wireless networks," IEEE Network, vol. 33, no. 4, pp. 6-8, 2019.

[7] M. Seyedebrahimi, A. Raschella, F. Bouhafs, M. Mackay, Q. Shi, and M. H. Eiza, "A centralised Wi-Fi management framework for D2D communications in dense Wi-Fi networks," in 2016 IEEE Conference on Standards for Communications and Networking (CSCN), pp. 1-6, Berlin, Germany, 2016.

[8] G. C. Deepak, F. Bouhafs, A. Raschellà, M. Mackay, and Q. Shi, "Radio resource management framework for energy-efficient communications in the Internet of Things," Transactions on Emerging Telecommunications Technologies, vol. 30, no. 12, 2019.

[9] A. Raschellà, F. Bouhafs, M. Mackay et al., "A dynamic access point allocation algorithm for dense wireless LANs using potential game," Computer networks, vol. 167, 2020.

[10] N. T. Somantri and I. Iskandar, "Asymmetric carrier aggregation on Lte-advanced access networks," in 2018 12th Interna- tional Conference on Telecommunication Systems, Services, and Applications (TSSA), pp. 4-5, Yogyakarta, Indonesia, 2018.

[11] F. Bouhafs, M. Seyedebrahimi, A. Raschella, M. Mackay, and Q. Shi, "Per-flow radio resource management to mitigate interference in dense IEEE 802.11 wireless LANs," IEEE Transaction on Mobile Computing, vol. 19, no. 5, pp. 1170$1183,2020$.

[12] O. Galinina, A. Pyattaev, S. Andreev, M. Dohler, and Y. Koucheryavy, "5G multi-RAT LTE-WiFi ultra-dense small cells: performance dynamics, architecture, and trends," IEEE Journal on Selected Areas in Communications, vol. 33, no. 6, pp. 1224-1240, 2015.

[13] R. Zhang, M. Wang, L. X. Cai, Z. Zheng, X. Shen, and L. L. Xie, "LTE-unlicensed: the future of spectrum aggregation for cellular networks," IEEE Wireless Communications, vol. 22, no. 3, pp. 150-159, 2015.

[14] R. Ali, B. Kim, S. W. Kim, H. S. Kim, and F. Ishmanov, "(ReLBT): a reinforcement learning-enabled listen before talk mechanism for LTE-LAA and Wi-Fi coexistence in IoT," Computer Communications, vol. 150, pp. 498-505, 2020.

[15] J. Zhang, S. Liu, R. Yin, G. Yu, and X. Jin, "Coexistence algorithms for LTE and WiFi networks in unlicensed spectrum: performance optimization and comparison," Wireless Networks, vol. 27, no. 3, pp. 1875-1885, 2021.

[16] Z. Qin, A. Li, and H. Wang, "Modeling and performance analysis of LTE coexisting with Wi-Fi," in 2020 IEEE 8th International Conference on Information, Communication and Networks (ICICN), pp. 43-50, Xi'an, China, 2020.

[17] M. Ali, S. Qaisar, M. Naeem, W. Ejaz, and N. Kvedaraite, "LTE-U WiFi HetNets: enabling spectrum sharing for 5G/beyond 5G systems," IEEE Internet of Things Magazine, vol. 3, no. 4, pp. 60-65, 2020.

[18] D. D. Nguyen, H. X. Nguyen, and L. B. White, "Evaluating performance of RAT selection algorithms for $5 \mathrm{G}$ Hetnets," IEEE Access, vol. 6, pp. 61212-61222, 2018.

[19] A. Raschella, F. Bouhafs, G. C. Deepak, and M. Mackay, "QoS aware radio access technology selection framework in heterogeneous networks using SDN," Journal of Communications and Networks, vol. 19, no. 6, pp. 577-586, 2017.

[20] E. Ibarrola, M. Davis, C. Voisin, C. Close, and L. Cristobo, "QoE enhancement in next generation wireless ecosystems: a machine learning approach," IEEE Communications Standards Magazine, vol. 3, no. 3, pp. 63-70, 2019.

[21] E. Coronado, S. N. Khan, and R. Riggio, "5G-EmPOWER: a software-defined networking platform for $5 \mathrm{G}$ radio access networks," IEEE Transactions on Network and Service Management, vol. 16, no. 2, pp. 715-728, 2019.

[22] F. Bouhafs, M. Mackay, A. Raschella et al., "Wi-5: a programming architecture for unlicensed frequency bands," IEEE Communications Magazine, vol. 56, no. 12, pp. 178-185, 2018.

[23] A. Raschellà and A. Umbert, "Implementation of Cognitive Radio Networks to evaluate spectrum management strategies in real-time," Computer Communications, vol. 79, pp. 37-52, 2016.

[24] G. P. Tank, A. Dixit, A. Vellanki, and D. Annapurna, "Software-defined networking: the new norm for networks," White Paper, Palo Alto, CA, USA, 2012.

[25] "Cisco ACI and OpFlex connectivity for orchestrators," September 2020, https://www.cisco.com/c/en/us/td/docs/ switches/datacenter/aci/apic/sw/kb/b_Cisco_ACI_and_ OpFlex_Connectivity_for_Orchestrators.pdf. 
[26] O. Aldhaibani, Developing an SDWN Architecture for Wireless Network Engineering to Support a Quality of Experience Aware Handover, [Ph.D. thesis], Dept. of Computer Science, LJMU, Liverpool, UK, 2019, http://researchonline.ljmu.ac.uk/id/ eprint/11779/.

[27] O. Aldhaibani, F. Bouhafs, M. Makay, and A. Raschellá, "An SDN-based architecture for smart handover to improve QoE in IEEE 802.11 WLANs," in 2018 32nd International Conference on Advanced Information Networking and Applications Workshops (WAINA), pp. 287-292, Krakow, Poland, 2018.

[28] 3GPP specification Technical Report (TR) 38.211, "NR; physical channels and modulation," Rel, vol. 16, 2020.

[29] K. Sood, S. Liu, S. Yu, and Y. Xiang, "Dynamic access point association using software defined networking," in 2015 International Telecommunication Networks and Applications Conference (ITNAC), pp. 226-231, Sydney, NSW, Australia, 2015.

[30] S. Agrawal, A. Das, A. Gaikwad, and S. Dhage, "Customer churn prediction modelling based on behavioural patterns analysis using deep learning," in 2018 International Conference on Smart Computing and Electronic Enterprise (ICSCEE), pp. 1-6, Shah Alam, Malaysia, 2018. 